\title{
The Physical Modification of a Natural Rubber-Polypropylene Thermoplastic Elastomer Blend by Azobisformamide Blowing Agent
}

\author{
Safia Merabet, Farid Riahi, and Abdelmalek Douibi \\ Laboratoire de Préparation, Modification et Application des Matériaux Polymériques Multiphasiques, Université Ferhat Abbas, \\ 19000 Sétif, Algeria \\ Correspondence should be addressed to Farid Riahi, fariahi38@gmail.com
}

Received 29 April 2012; Accepted 5 June 2012

Academic Editors: H. M. da Costa, B. Hazer, A. Mousa, and C. T. Ratnam

Copyright () 2012 Safia Merabet et al. This is an open access article distributed under the Creative Commons Attribution License, which permits unrestricted use, distribution, and reproduction in any medium, provided the original work is properly cited.

The decomposition of azobisformamide (ABFA) blowing agent and its expansion in a soft grade of thermoplastic natural rubber composed of a 70/30 natural rubber (NR)/polypropylene (PP) was monitored using a Haake plasticorder. The aim of the study was to evaluate the effect of different concentrations of the blowing agent and to investigate its eventual interactions with a sulfurbased curing system that was used for the dynamic vulcanization. The plastograms allowed to detect variations in the initial mixing torque as well as the level of the final equilibrium torque for both unvulcanized and dynamically vulcanized blends. The plastograms of the dynamically vulcanized blends were characterized by the appearance of a second peak that corresponds to the crosslinking reaction which followed the blowing agent decomposition reaction. Since changing the order of the addition of the curatives with respect to the blowing agent did not cause any major effect on the general trend of the plastograms, it was concluded that the azobisformamide blowing agent did not alter the chemical action of the curatives and vice versa. The effects on the plastograms caused by variations in the blowing agent concentration were also reflected through the density measurements.

\section{Introduction}

Thermoplastic elastomers (TPE) are considered as a unique class of materials which play a great role in the polymer industry because they combine the advantages of the processing of thermoplastics with the outstanding mechanical properties and elastic recovery of vulcanized rubbers [1]. Different types of TPEs have been developed. Among them, those prepared by melt mixing natural rubber (NR) with a polyolefin such as polypropylene (PP) and which are classified as thermoplastic natural rubber (TPNR) have gained more acceptance because of the ease of preparation [2]. Research on TPNR is very extensive and can be summarized in three main categories which are the development of different types of TPNR by varying blend components [3$5]$, the investigation of different crosslinking agents for the dynamic vulcanization [6-8], and study of the effects of the chemical modification of the NR and/or the polyolefin part in the blend [9-11]. These chemical modifications can enhance blend component compatibilization and improve some useful properties of the material. But it is also possible to physically modify TPNRs through the use of blowing agents. Blowing agents are substances that give up a great amount of gas or vapor resulting in a cellular structure. The resulting cellular structure of the material depends not only on the type and concentration of the blowing agent but also on the operating conditions. On the other hand, many different techniques have been reported for the evaluation of solid chemical blowing agents [12], but they mainly refer to their decomposition temperature and gas volume in the pure state. In addition, these studies concern only single phase polymeric matrices. The aim of this work is therefore to investigate the effect of the addition of a chemical blowing agent, namely, azobisformamide (ABFA), on the physical properties of a 70/30 NR/PP thermoplastic elastomer blend. The objective of the study is to monitor the blowing agent decomposition reaction using a Haake plasticorder and to evaluate possible interactions between the blowing agent and 
TABLE 1: Composition of the blends.

\begin{tabular}{lcccc}
\hline \multirow{2}{*}{ Ingredient } & \multicolumn{5}{c}{$\begin{array}{l}\text { Designation (quantities in parts per hundred } \\
\text { parts by weight of rubber) }\end{array}$} \\
& M1 & M2 & M3 & M4 \\
\hline NR & 70 & 70 & 70 & 70 \\
PP & 30 & 30 & 30 & 30 \\
ABFA & - & $0.4-1$ & $0.4-1$ & $0.4-1$ \\
Sulfur & - & - & 1 & - \\
TMTD & - & - & 1 & 1 \\
ZnO & - & - & 5 & - \\
Stearic acid & - & - & 1 & - \\
\hline
\end{tabular}

the sulfur-based curing system which is used for the dynamic vulcanization.

\section{Experimental}

2.1. Materials. The two main polymers used in this study, natural rubber and polypropylene were of commercial grade and were supplied by ENPC, Algeria. Natural rubber was an SMR 20 grade with a density of $0.93 \mathrm{gr} / \mathrm{cm}^{3}$, polypropylene used was an injection grade with a density of $0.91 \mathrm{gr} / \mathrm{cm}^{3}$ and a Melt Flow Index $\left(230^{\circ} \mathrm{C}, 2,16 \mathrm{Kg}\right)$ of $6.0 \mathrm{gr} / 10 \mathrm{~min}$.

The blowing agent used is azobisformamide that was used in a powder form and was supplied by SYNSET, Algeria. It has a molecular weight of $116 \mathrm{gr} / \mathrm{mole}$ and a heat of combustion of $225 \mathrm{Kcal} /$ mole at a decomposition temperature of $200^{\circ} \mathrm{C}$.

The chemicals used for the dynamic vulcanization, namely, sulfur, the crosslinking agent, TMTD (Tetra methyl thiuram disulfide) as an accelerator, and sulfur donor, $\mathrm{ZnO}$ (Zinc oxide), and stearic acid as activators were standard materials used in rubber formulations and were supplied by ENPC, Algeria.

2.2. Blend Preparation. The compositions of the blends in parts by weight of rubber (phr) are shown in Table 1. In this study all the NR/PP blends contained 70/30 rubber to plastic ratio but the blowing agent concentration was varied between 0.4 and $1 \mathrm{phr}$. To investigate the effect of the type of the crosslinking system, TMTD without sulfur in formulations M4 was used as a sulfur donor.

Premixing of natural rubber in the form of small pieces with polypropylene granulates was carried out in a laboratory single screw extruder with a length to diameter ratio of 20. Extrusion was performed at a screw speed of $30 \mathrm{rpm}$ and temperatures set at $170^{\circ} \mathrm{C}, 190^{\circ} \mathrm{C}$, and $200^{\circ} \mathrm{C}$ for the three barrel zones, respectively. After cooling at room temperature, the blend extrudates were grinded.

It is to be noted that for expanded compositions, a small quantity of Di-2-ethylhexyl phthalate plasticizer was used to ensure a good wetting of the blowing agent powder onto the material.

2.3. Blowing Agent Decomposition. A Haake plasticorder, model Rheocord 600 TARKAN, was used to monitor the evolution of the azobisformamide decomposition and expansion through the NR/PP thermoplastic elastomer blend. The mixing chamber with a cam-type mixer was set at $200^{\circ} \mathrm{C}$ and the rotor speed at $40 \mathrm{rpm}$.

After loading the mixer with 55 grams of each formulation the mixing torque of the Haake plasticorder was recorded continuously with time.

2.4. Physical Testing. To verify the effect of the decomposition and expansion of the ABFA blowing agent, the density of the different blends was measured using a pycnometer. In this case the samples were prepared using a melt flow indexer fitted with a die having a diameter of $2.09 \mathrm{~mm}$ and a length of $8 \mathrm{~mm}$. The samples were extruded at $200^{\circ} \mathrm{C}$ under a load of $5 \mathrm{Kg}$.

2.5. Swelling Index. In order to characterize the extent of crosslinking for the dynamically vulcanized blends, the swelling index was measured on swollen specimens after immersion in cyclohexane. The test specimens, which were obtained using the melt flow indexer, were weighed and then immersed in cyclohexane during 24 hours at room temperature. Prior to being weighed again the swollen samples were blotted with a filter paper in order to remove the superficial solvent. The swelling index was calculated as the ratio of the swollen mass to the initial mass.

\section{Results and Discussion}

3.1. Plastograms of NR and PP. The Haake rheometer torque-time curves, which will be referred to as plastograms, for neat NR and that of neat PP when used alone as well as their blend at a 70/30 NR/PP ratio are shown in Figure 1. These curves are characterized by an initial sharp rise in the torque followed by a milder steady decrease before reaching a final leveling off. This is explained by the fact that as the polymer, either NR or PP, is introduced into the mixing chamber in the solid state the force exerted on the rotors is maximal corresponding to a high torque value. Then, as the material starts to fuse under the shearing action of the rotors the torque decreases steadily. The final leveling off is an indication that complete homogenization of the polymer has been reached.

These plastograms show also that the resisting torque of NR is higher than that of PP. This could be attributed to the higher molecular weight of natural rubber compared to that of polypropylene. On the other hand, blending these two polymers gave rise to an intermediate behavior.

3.2. Effect of the Blowing Agent. In order to determine the appropriate concentration of the azobisformamide blowing agent which would give an optimum degree of expansion without exceeding a critical value beyond which the resulting cellular structure would collapse, two concentrations were initially tested, namely, a concentration of $2 \mathrm{phr}$, considered as the upper limit and a concentration of 1 phr as a conventionally practical one. In this context, the plastograms of PP with ABFA are shown in Figure 2. It is shown that the compositions containing the blowing agent exhibited a higher 


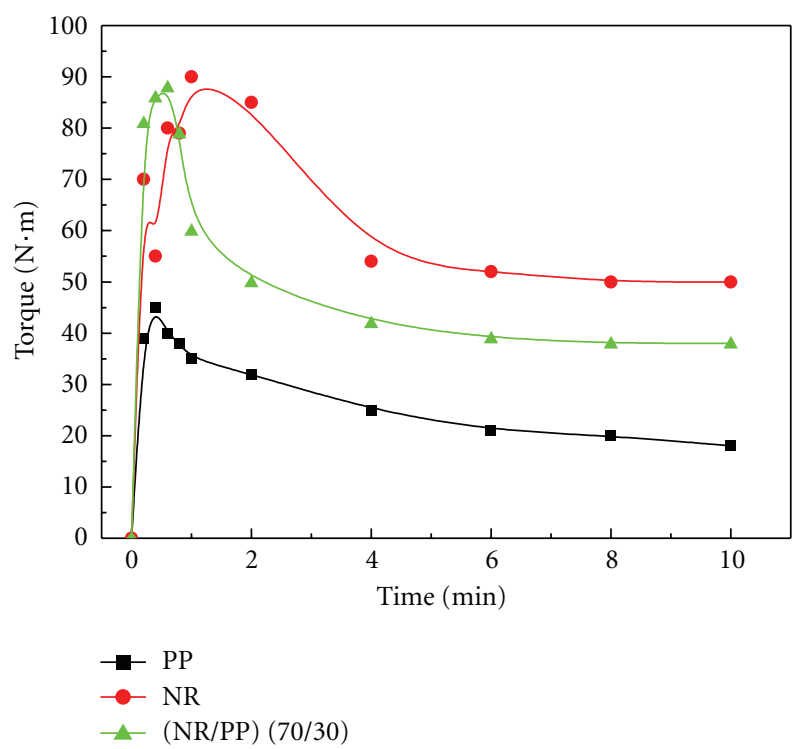

Figure 1: Plastograms of neat PP, neat NR, and NR/PP 70/30 blend.

value of the initial maximum torque. This could be attributed to the pressure exerted by the gases produced as a result of the decomposition of azobisformamide. It is also shown that using a concentration of $2 \mathrm{phr}$ caused an important decrease in the viscosity as manifested by the extent of the decrease in the torque. This is due to the exothermic nature of the blowing agent decomposition reaction which caused also the time of the onset of fusion to be reduced and the time to reach equilibrium to be prolonged.

Similarly, the plastograms of NR mixed with ABFA are shown in Figure 3. These plastograms reflect a different reactivity of NR with ABFA compared to that observed with PP. In fact, the addition of the blowing agent at both concentrations seemed to have decreased much the viscosity which is manifested by the important decrease in the torque. Moreover, the addition of a higher concentration of ABFA reduced the fusion time, as previously observed with PP, but a higher value of the initial torque was observed. This could be explained by the fact that the decomposition of this amount of the blowing agent resulted in a subsequent amount of gases which remained trapped inside the elastic polymeric matrix.

3.3. Effect of the Concentration of ABFA. The plastograms of the unvulcanized 70/30 NR/PP blends mixed with different concentrations of ABFA are shown in Figure 4. These curves show that as the amount of the blowing agent is increased from $0.4 \mathrm{phr}$ to $1 \mathrm{phr}$, the value of the maximum initial torque decreases and so does the level of the final torque.

For the soft grades of TPNR where the rubber content is higher than $65 \%$ its partial crosslinking is still required. The addition of a small concentration of a crosslinking system during melt mixing, a process called the dynamic vulcanization, has been proven to offer an efficient route to prepare thermoplastic elastomers with satisfactory properties [13]. It is therefore necessary to investigate the effect of the

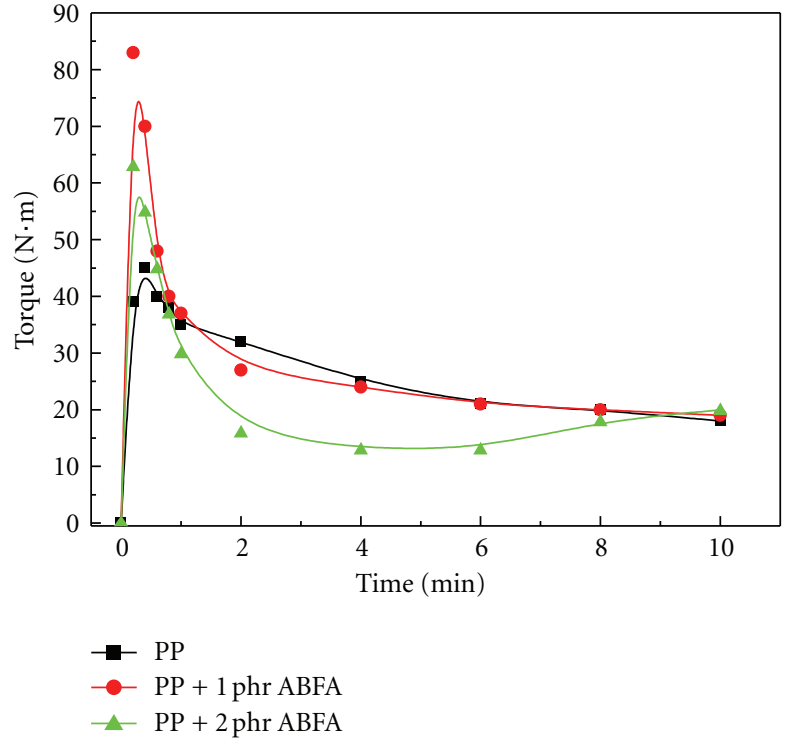

FIGURe 2: Plastograms of neat PP and PP mixed with two concentrations of ABFA.

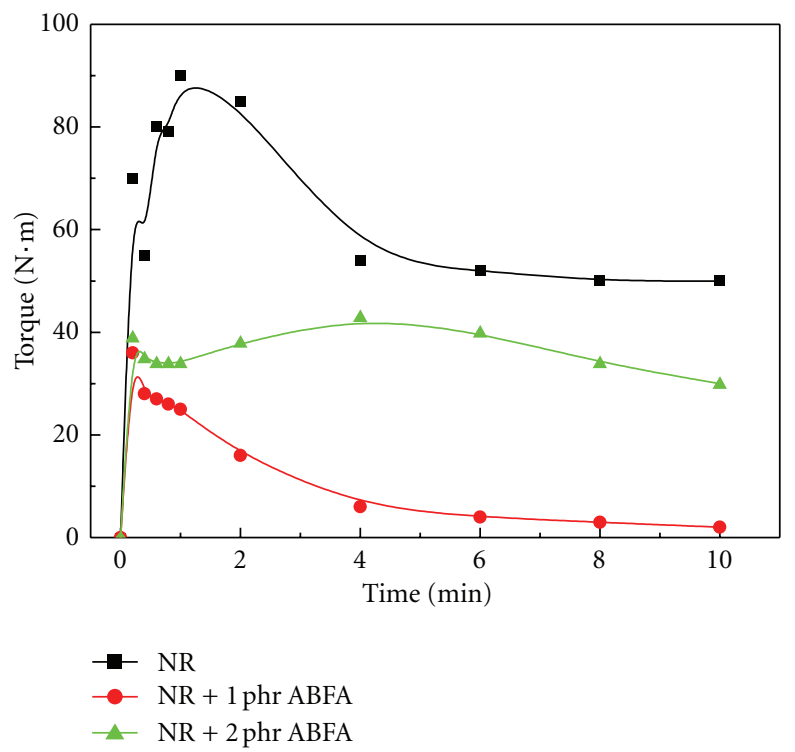

FIGURE 3: Plastograms of neat NR and NR mixed with two concentrations of ABFA.

crosslinking agent and its eventual interaction with the blowing agent. Figure 5 presents the plastograms of the ABFAcontaining blends which were dynamically vulcanized with a sulfur-based crosslinking system. The basic distinction of these curves with respect to those of the unvulcanized blends is the appearance of a second peak that corresponds to the onset of the crosslinking reaction which followed the blowing agent decomposition reaction. It is also observed that the value of the initial maximum torque for the dynamically vulcanized blends was reduced but a higher final level of the torque was reached. This could be attributed to the formation of interchain crosslinks which acted as a barrier against 


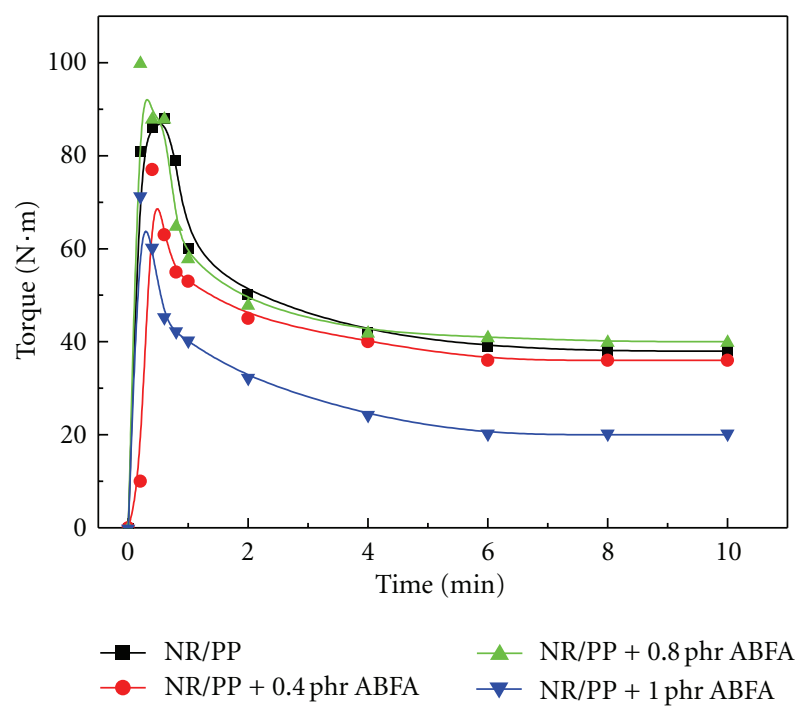

FIGURE 4: Plastograms of unvulcanized NR/PP blends mixed with different concentrations of ABFA.

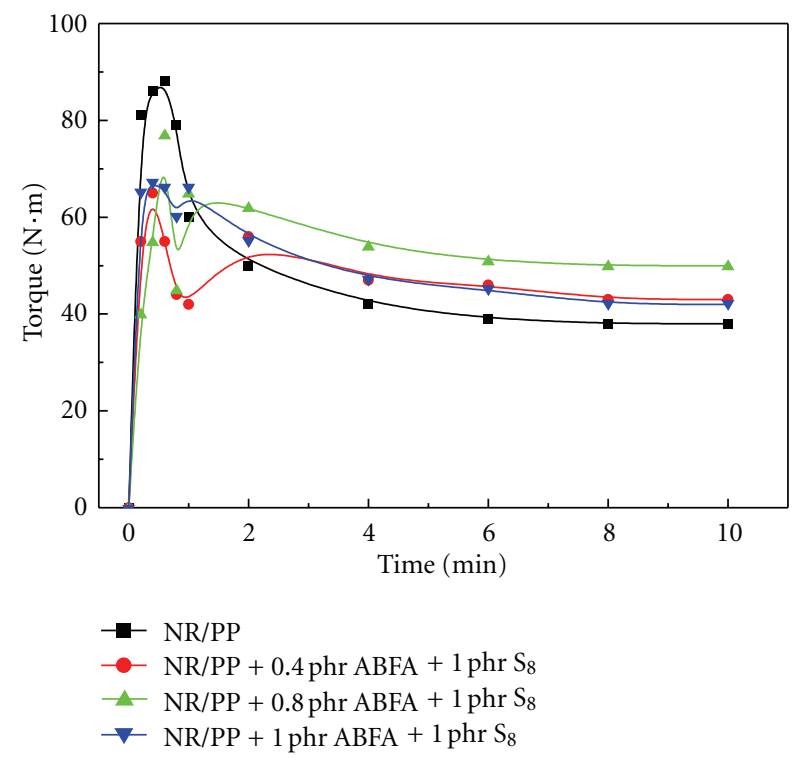

FIGURe 5: Plastograms of the dynamically sulfur-vulcanized NR/PP blends mixed with different concentrations of ABFA.

the escape of the decomposition gases out of the polymeric matrix. The higher final level of the torque is an indication of a more stable network.

3.4. The Effect of the Type of the Crosslinking Agent. The type of the crosslinking agent used for the dynamic vulcanization is as important as its concentration. The stability of the crosslinks formed depends much on the proper choice of the crosslinking agent used. For instance, TMTD, when used alone, can act as an efficient crosslinking agent and would produce monosulfidic crosslinks that are more stable than the polysulfidic crosslinks which are formed when sulfur is used instead.

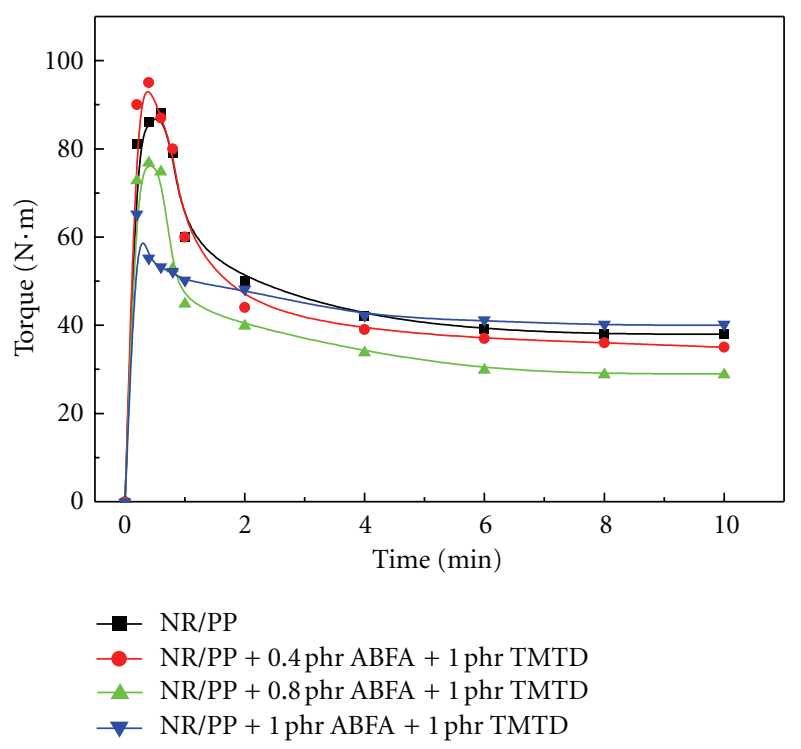

FIGURE 6: Plastograms of the dynamically TMTD-vulcanized NR/PP blends mixed with different concentrations of ABFA.

TABLE 2: Swelling index for the different vulcanizates.

\begin{tabular}{lccc}
\hline Ingredient & \multicolumn{3}{c}{$\begin{array}{c}\text { Composition (quantities in parts per hundred } \\
\text { parts (phr) by weight of rubber) }\end{array}$} \\
\hline $\mathrm{NR}$ & 70 & 70 & 70 \\
$\mathrm{PP}$ & 30 & 30 & 30 \\
$\mathrm{ABFA}$ & 0.4 & 0.8 & 0.8 \\
Sulfur & 1 & 1 & - \\
TMTD & 1 & - & 1 \\
ZnO & 5 & 5 & - \\
Stearic acid & 1 & 1 & - \\
Swelling index & $\mathbf{2 . 3 5 7 1}$ & $\mathbf{1 . 7 2 1 0}$ & $\mathbf{2 . 6 7 7 3}$ \\
\hline
\end{tabular}

As shown in Figure 6, in contrast to the NR/PP blends blown with ABFA and which were dynamically vulcanized with sulfur and accelerators, those containing TMTD did not exhibit a crosslinking peak. This means that if such a crosslinking could not take place along with the decomposition of the blowing agent, then TMTD which is supposed to act as a sulfur donor, did not contribute in the dynamic vulcanization. In order to verify whether TMTD actually crosslinked the material, the swelling index for the different vulcanizates was measured and the results are shown in Table 2. These results show that the swelling index of the blends dynamically vulcanized with sulfur is lower than that of the blends cured with TMTD alone. Since the restriction to swelling increases as the degree of crosslinking is increased, therefore these results suggest that TMTD at a concentration of $1 \mathrm{phr}$ did not vulcanize this TPNR.

3.5. Effect of the Order of the Addition of Additives. To detect any eventual interaction that could take place between azobisformamide, the blowing agent and the curing system composed of sulfur, accelerator, and activators, these additives were added to the 70/30 NR/PP blend according to two 


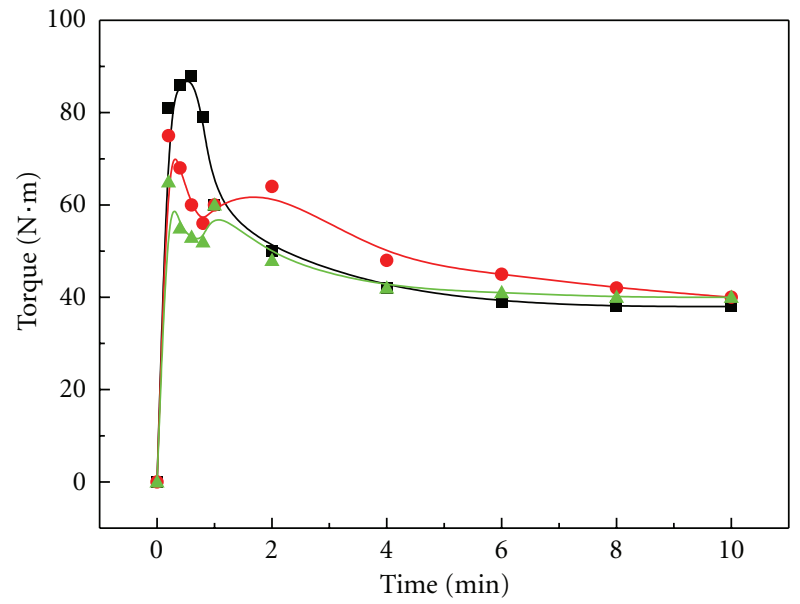

- NR/PP
- - Bulk-mixing
- Step-mixing

Figure 7: Plastograms of the dynamically sulfur-vulcanized NR/PP blends mixed with 1 phr of ABFA according to the bulk-mixing method and the step-mixing method.

different methods. In the first method which will be referred to as the bulk-mixing method, the blowing agent as well as the curatives were all mixed together before adding them in bulk to the blend. But in the second method which will be referred to as the step-mixing method, the curatives are added first after the fusion of the blend, then in a third step the blowing agent is added separately after 1 minute of mixing.

The plastograms obtained according to these two methods are shown in Figure 7. We can see that when the sulfur and accelerators are added before the blowing agent according to the step-mixing method the torque slightly increases compared to the peak with the bulk-mixing method. Also, except for a slight decrease of the level of the final torque, the same general trend is shown for the blend tested according to the bulk-mixing method. This suggests that the blowing agent did not alter the action of the curatives and vice versa.

3.6. Density. During the blowing process the cells are formed when the saturation limits of the gases liberated are reached [14]. This was manifested in the Haake curves as the steady decrease in the final torque which represents the end of the growth of the cells. At this point a major portion of the liberated gases remain within the polymeric matrix inside the pores. It is therefore reasonable to evaluate the efficiency of the blowing agent through the measurements of density as the main physical property that is affected by the blowing agent.

Figure 8 shows the variation of the extrudate density with the blowing agent concentration. According to these bar graphs the density decreases with increasing the amount of ABFA except for the concentrations of $0.6 \mathrm{phr}$ and $1 \mathrm{phr}$. The lowest decrease in the density which is estimated to be $14 \%$ was obtained at a concentration of $0.4 \mathrm{phr}$ and $0.8 \mathrm{phr}$. The

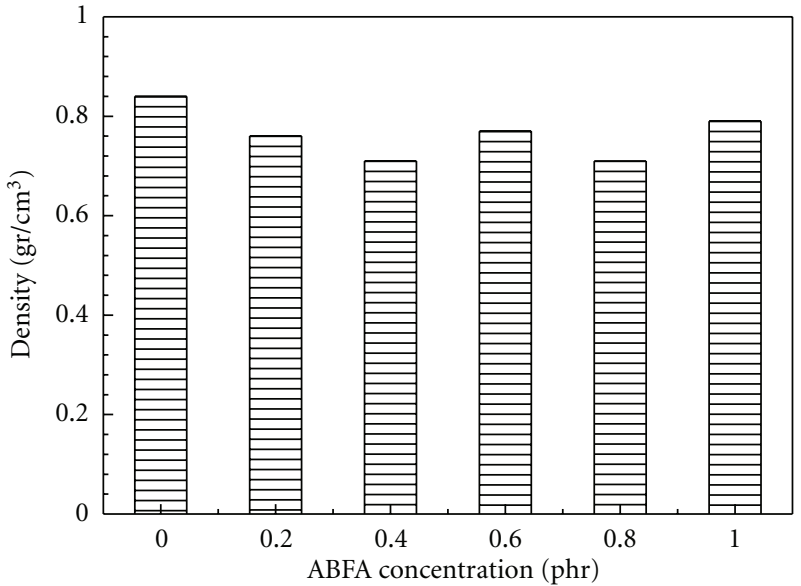

FIGURE 8: Variation of the density with the blowing agent concentration for a dynamically sulfur-vulcanized 70/30 NR/PP blend.

fluctuations could be attributed to the sensitivity of the test or to the fact that there is an optimum concentration above which the volume of the gases exceeds its saturation level hence part of the liberated gases escape out of the matrix. It is therefore important to use an optimum concentration of the blowing agent in order to produce a uniform and stable cellular structure.

\section{Conclusion}

In this study a Haake plasticorder was used to evaluate the decomposition of azobisformamide blowing agent within a 70/30 NR/PP thermoplastic elastomer blend. The influence of various parameters was investigated. The plastograms of NR and PP alone reflected different reactivities of the blowing agent with the polymeric matrix with respect to the initial torque peak as well as the level of the final mixing torque. The plasticorder detected also these effects with the 70/30 NR/PP blends blown with different concentrations of the blowing agent. The plastograms of the dynamically vulcanized blends showed the appearance of a second peak that corresponds to the crosslinking reaction which followed the blowing agent decomposition reaction. It was also found that when using TMTD alone, no crosslinking took place compared to the case where sulfur and accelerators were used for the dynamic vulcanization. This result was confirmed through the swelling index measurements. Varying the order of the addition of the curatives and the blowing agent allowed also to conclude that azobisformamide did not alter the chemical action of the sulfur-based crosslinking agent and vice versa. Increasing the blowing agent concentration was also found to cause a subsequent decrease in the density. All these results demonstrated that an optimum concentration of ABFA should be used in order to obtain a stable cellular structure. In brief, the investigated parameters allowed to conclude that the use of the plasticorder is an efficient and reliable way to monitor a blowing agent decomposition within a polymeric matrix. 


\section{References}

[1] A. D. Thorn, Thermoplastic Elastomers, Rapra, Shawbury, UK, 1980.

[2] F. Riahi, D. Benachour, and A. Douibi, "Dynamically vulcanized thermoplastic elastomer blends of natural rubber and polypropylene," International Journal of Polymeric Materials, vol. 53, no. 2, pp. 143-156, 2004.

[3] M. Suryadiansyah and H. Ismail, "Thermoplastic elastomers based on polypropylene/natural rubber and polypropylene/recycle rubber blends," Polymer Testing, vol. 21, no. 4, pp. 389-395, 2002.

[4] A. Y. Coran and O. Chung, "The morphology of rubber/plastic blends," Rubber Chemistry and Technology, vol. 70, no. 5, pp. 781-797, 1997.

[5] P. V. Ashalata, K. E. George, and D. J. Francis, "Scanning electron microscopic studies of PP/EPDM/NR ternary blends," Journal of Elastomers and Plastics, vol. 29, no. 1, pp. 92-101, 1997.

[6] D. J. Elliot, "Influence of crosslinking agents and interfacial adhesion promoters on the properties of natural rubberpolypropylene blends," Rubber Developments, vol. 39, no. 3-4, 1987.

[7] Z. Ghazali, A. F. Johnson, and K. Z. Dahlan, "Radiation crosslinked thermoplastics natural rubber (TPNR) foams," Radiation Physics and Chemistry, vol. 55, no. 1, pp. 73-79, 1999.

[8] U. S. Ishiaku, A. Shaharum, Z. A. M. Ishak, and H. Ismail, "Thermo-oxidative ageing of poly(vinyl chloride) based thermoplastic elastomers: effect of "new" epoxidized natural rubber," KGK-Kautschuk und Gummi Kunststoffe, vol. 50, no. 4, pp. 292-298, 1997.

[9] U. S. Ishiaku, A. Shaharum, H. Ismail, and Z. A. M. Ishak, "The effect of an epoxidized plasticizer on the thermooxidative ageing of poly(vinyl chloride)/epoxidized natural rubber thermoplastic elastomers," Polymer International, vol. 45, no. 1, pp. 83-91, 1998.

[10] H. Ismail and S. Suzaimah, "Styrene butadiene rubber/epoxidized natural rubber blends: dynamic properties, curing characteristics and swelling studies," Polymer Testing, vol. 19, pp. 879-888, 2000.

[11] A. Mousa, U. S. Ishiaku, and Z. A. M. Ishak, "Rheological and viscoelastic behavior of dynamically vulcanized poly(vinyl chloride)-epoxidized natural-rubber thermoplastic elastomers," Journal of Applied Polymer Science, vol. 74, no. 12, pp. 2886-2893, 1999.

[12] A. Douibi, D. Benachour, F. Mansouri, D. Melab, and A. Benmekideche, "Effect of the addition of chemical blowing agents on the processability of a rigid PVC compound," International Journal of Polymeric Materials, vol. 52, no. 10, pp. 917-925, 2003.

[13] N. R. Legge, G. Holden, and H. E. Shroeder, Thermoplastic Elastomers: A Comprehensive Review, Hanser Publishers, New York, NY, USA, 1987.

[14] F. Riahi, R. Doufnoune, and C. Bouremel, "Subtitution of pentane by a mineral clay as a blowing agent for phenolic foam," International Journal of Polymeric Materials, vol. 56, no. 9, pp. 929-938, 2007. 

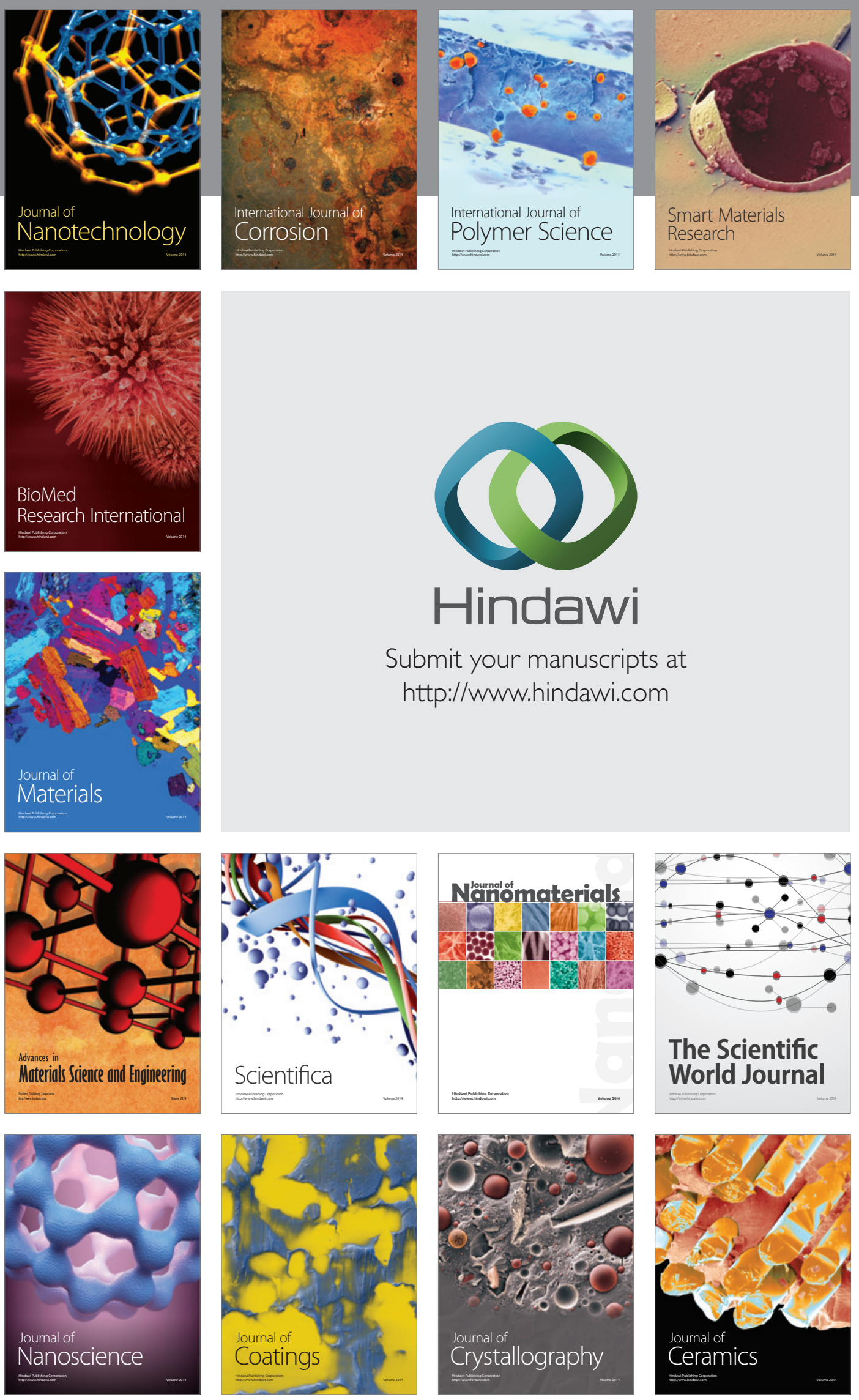

The Scientific World Journal

Submit your manuscripts at

http://www.hindawi.com

\section{World Journal}

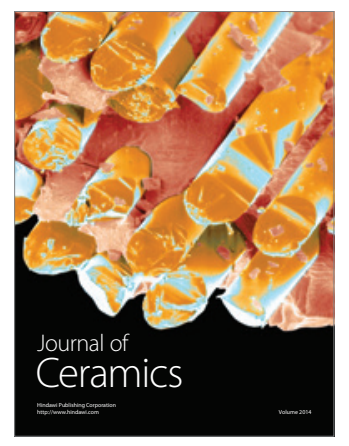

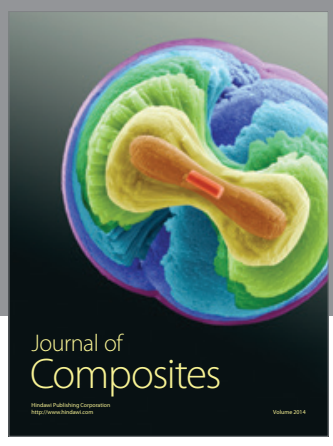
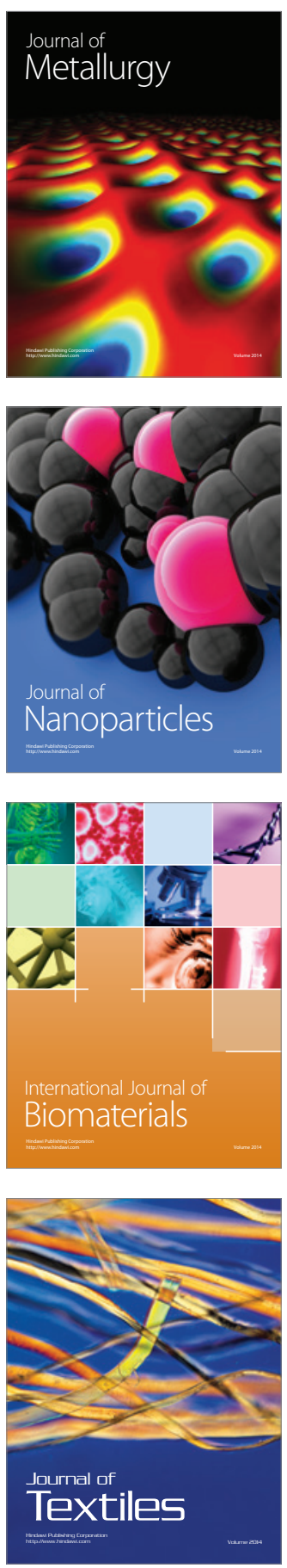